\title{
Human cathelicidin antimicrobial peptide suppresses proliferation, migration and invasion of oral carcinoma HSC -3 cells via a novel mechanism involving caspase- 3 mediated apoptosis
}

\author{
${\text { XI } \text { CHEN }^{1,2^{*}} \text {, SHENYING JI }}^{1,2^{*}}$, JIA SI $^{1,2}$, XIANGYU ZHANG ${ }^{1,2}$, \\ XIAOYAN WANG ${ }^{3}$, YONG GUO ${ }^{3}$ and XIANQIONG ZOU ${ }^{1-3}$ \\ ${ }^{1}$ Laboratory of Mucosal Immunology, Affiliated Stomatology Hospital of Guilin Medical University, Guilin, \\ Guangxi 541004; ${ }^{2}$ College of Basic Medicine, Guilin Medical University, Guilin, Guangxi 541100; \\ ${ }^{3}$ College of Biotechnology, Guilin Medical University, Guilin, Guangxi 541004, P.R. China
}

Received March 10, 2020; Accepted October 8, 2020

DOI: $10.3892 / \mathrm{mmr} .2020 .11629$

\begin{abstract}
Human cathelicidin antimicrobial peptide and its active product, LL-37 (CAMP/LL-37), exhibit a broad spectrum of antimicrobial effects. An increasing number of studies have shown that human CAMP/LL-37 also serves significant roles in various types of cancer. The primary aims of the present study were to investigate the roles and mechanisms of human CAMP/LL-37 in oral squamous cell carcinoma (OSCC) cells. The results indicated that either LL-37 C-terminal deletion mutants (CDEL) or CAMP stable expression in HSC-3 cells reduced colony formation, proliferation, migration and invasion ability of the cells. Expression analysis demonstrated that either CDEL or CAMP stable expression in HSC-3 cells induced caspase-3 mediated apoptosis via the $\mathrm{P} 53-\mathrm{Bcl}-2 / \mathrm{BAX}$ signalling pathway, whereas the levels of cell cycle-related proteins, cyclin B1 and PKR-like ER kinase, were significantly upregulated in the CAMP, but not in the CDEL overexpressing cells. Transcriptional profile comparisons revealed that CDEL or CAMP stable expression in HSC-3 cells upregulated expression of genes involved in the IL-17-dependent pathway compared with the control. Taken together, these results suggest that CAMP may act as a tumour suppressor in OSCC cells, and the underlying mechanism
\end{abstract}

Correspondence to: Dr Xianqiong Zou, Laboratory of Mucosal Immunology, Affiliated Stomatology Hospital of Guilin Medical University, 109 Huanchengbeier Road, Guilin, Guangxi 541004, P.R. China

E-mail: xianqiongzou2009@yahoo.com

${ }^{*}$ Contributed equally

Key words: CAMP, oral squamous cell carcinoma, tumour suppressor, apoptosis, LL-37 involves the induction of caspase-3 mediated apoptosis via the P53-Bcl-2/BAX signalling pathway.

\section{Introduction}

Human cathelicidin antimicrobial peptide (CAMP) and its active product, LL-37, serve important roles in infectious diseases (including viral, bacterial and fungal infections) and autoimmune diseases (1-5). Previously, it has been found that CAMP/LL-37 also has a significant effect on the initiation and progression of various types of cancer (6-9). Additionally, it has been indicated that the upregulation of CAMP/LL-37 expression contributes to its tumorigenic effect in breast cancer, ovarian cancer, lung cancer, prostate cancer, pancreatic cancer, malignant melanoma and skin squamous cell carcinoma (10-16). Moreover, the downregulation of CAMP/LL-37 expression contributes to its anticancer effects on colon cancer, gastric cancer and haematological malignancies (17-21). The LL-37-induced activation of membrane receptors and subsequent signalling pathways leads to the alteration of cellular functions (6-9). Different membrane receptors on various cancer cells appear to be responsible for the tissue-specific effects of LL-37 (6-9).

hCAP18 ${ }_{109-135}$, a 27 amino acids peptide in the C-terminal of hCAP18, induces caspase-independent apoptosis of oral squamous cell carcinoma (OSCC) SAS-H1 cells (22). KI-21-3, a shortened fragment of LL-37, exhibits considerable oncolytic properties on SCC-4 carcinoma cells via the antiproliferative and caspase-3 dependent apoptotic pathway (23). A previous study by the same authors also demonstrated that the expression levels of CAMP/LL-37 were downregulated significantly in OSCC tissues (24), suggesting that CAMP exerts an inhibitory effect on the initiation and progression of OSCC, although the underlying mechanisms remain unclear in OSCC.

The aim of the present study was to investigate the roles and mechanisms of action of human CAMP/LL-37 in OSCC HSC-3 cells $(25,26)$ using a colony-formation, CCK-8, wound healing and Transwell invasion assays, as well as expression analysis on the basis of established LL-37 C-terminal deletion 
mutants (CDEL) and CAMP stable overexpression in OSCC HSC-3 cell lines.

To the best of our knowledge, the results of the present study are the first to determine the potential mechanism underlying the effects of the overexpression of human CAMP in OSCC HSC- 3 cells.

\section{Materials and methods}

Plasmid construction. High-fidelity Herculase enzyme (Agilent Technologies, Inc.) was used for amplification of cDNA from foetal brain tissue (Clontech Laboratories) and was used as the template. The primers used for the amplification of the open reading frame (ORF) of CAMP were as follows: Forward, 5'-CGGAATTCAATGGGGACCATGAAGACCCAAAGG-3' and reverse, 5'-CGGGATCCCTAGGACTCTGTCCTGGGTAC AAG-3'. The primers used for the amplification of the ORF of CDEL were as follows: Forward, 5'-CGG AATTCAATGGGG ACCATGAAGACCCAAAGG-3' and reverse, 5'-CGGGATC CCTAGGCAAATCTCTTGTTATCCTTATCACAAC-3'. The amplification conditions were as follows: Pre-denaturation, $95^{\circ} \mathrm{C}$ for $2 \mathrm{~min}$; followed by 40 cycles at $95^{\circ} \mathrm{C}$ for $20 \mathrm{sec}, 55^{\circ} \mathrm{C}$ for $20 \mathrm{sec}$ and $72^{\circ} \mathrm{C}$ for $45 \mathrm{sec}$; with a final extension step of $72^{\circ} \mathrm{C}$ for $3 \mathrm{~min}$. PCR products were purified and were then ligated in to pFlag-CMV4 (Sigma-Aldrich; Merck KGaA). Plasmid constructs were confirmed by sequencing (Sangon Biotech Co., Ltd.).

Cell culture. Human OSCC HSC-3 cells (ATCC) were cultured in RPMI-1640 medium (Gibco; Thermo Fisher Scientific, Inc.) supplemented with $10 \%$ FBS (Gemini Bio-Products). KB cells (ATCC) were maintained in modified Eagle's medium (Gibco; Thermo Fisher Scientific, Inc.) supplemented with 10\% FBS. The cells were incubated in an incubator at $37^{\circ} \mathrm{C}$ with $5 \% \mathrm{CO}_{2}$.

Generation of CDEL or CAMP stably expressing cells. HSC-3 cells were grown to $60-80 \%$ confluency, and transfected using Expressfect ${ }^{\mathrm{TM}}$ Transfection Reagent (Denville Scientific). Following $24 \mathrm{~h}$ of transfection, the transfected

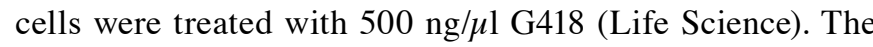
medium containing G418 was replaced every $48 \mathrm{~h}$. Cells were subsequently cultured with $200 \mathrm{ng} / \mu \mathrm{l}$ G418 in the medium to maintain cell resistance. Single positively transfected cell clones were screened using the filter paper method (27). The expression of either CDEL or CAMP was confirmed by western blot analysis and immunofluorescence (28-30).

Immunofluorescence. Stably transfected HSC-3 cells were fixed with cold methanol for $5 \mathrm{~min}$ at $-20^{\circ} \mathrm{C}$, then washed with DPBS, and permeabilized with $0.25 \%$ Triton X-100 for $10 \mathrm{~min}$. After blocking for $1 \mathrm{~h}$ with $1 \%$ BSA in DPBS/0.1\% Tween-20, and rinsing with DPBS three times, the cells were incubated with rabbit anti-cathelicidin (cat. no. ab69484; Abcam) or mouse anti-LL37 (cat. no. sc-166770; Santa Cruz Biotechnology, Inc.) antibodies at room temperature for $1 \mathrm{~h}$, followed by incubation with Alexa Fluor 488-conjugated goat anti-rabbit IgG or Alexa Fluor 594-conjugated goat anti-mouse IgG (ProteinTech Group, Inc.) at room temperature for $1 \mathrm{~h}(27,28)$. Nuclei were stained with $300 \mathrm{nM}$ DAPI at room temperature for $1 \mathrm{~min}$, and the cells were then imaged using an epifluorescence microscope (Nikon Eclipse Ti; Nikon Corporation; magnification, $\mathrm{x} 400)$.

Colony formation assay. Stably transfected HSC-3 cells were adjusted to 1,500 cells per dish. The solution was replaced every 3 days, and crystal violet staining was performed at room temperature for $3 \mathrm{~min} 12$ days later. Cells were imaged by camera. A group of $>50$ cells was considered as one colony. The number of clones was counted under a light microscope (magnification, x100) and 5 fields of view were analyzed.

Cell Counting Kit-8 (CCK-8) assay. A CCK-8 assay (Dojindo Molecular Technologies, Inc.) was used to measure cell viability. Stably transfected HSC-3 cells $\left(9 \times 10^{3}\right.$ cells/well) were seeded into a 24-well plate. Viability was assessed after $0,24,48$ and $72 \mathrm{~h}$ following complete adherence. A total of $100 \mu \mathrm{l}$ CCK- 8 reagent was added to each well of the first 24-well plate, covered with tin foil and placed in a cell incubator $\left(37^{\circ} \mathrm{C}, 5 \% \mathrm{CO}_{2}\right)$ for $2 \mathrm{~h}$, and subsequently the absorbance was measured at $450 \mathrm{~nm}$ using a microplate reader (BioTek Instruments, Inc.).

Wound healing assay. Stably transfected HSC-3 cells were seeded into $1.2 \times 10^{6}$ cells/well in a 6 -well plate, and cultured for $16 \mathrm{~h}$ to form a confluent monolayer. The confluent cultures were scratched with sterile $200-\mu 1$ pipette tips. The culture medium was removed, and washed three times with DPBS. After washing and removing the cell debris, the cells were cultured with serum-free RPMI medium, and the images were captured over time using a light microscope (IX-70; Olympus; magnification, $\mathrm{x}$ 40) and analysed using ImageJ version 1.8.0 software (National Institutes of Health).

Transwell invasion assay. The stably transfected HSC- 3 cells $\left(2.5 \times 10^{5} / \mathrm{ml}, 200 \mu \mathrm{l}\right)$ were seeded into a Matrigel-coated Transwell upper chamber (Corning, Inc.) in serum-free RPMI medium, and $\sim 750 \mu 1$ RPMI medium containing $20 \%$ serum was added to the lower chambers. The chambers were placed in the 24-well plate, and cultured in a cell incubator for $64 \mathrm{~h}$ and subsequently stained with haematoxylin at room temperature for $10 \mathrm{~min}$, and observed and imaged using a light microscope (magnification, x200).

Western blot analysis. Cells were washed with DPBS and then total protein was extracted using mammalian cell lysate buffer (Biyuntian Bio-Technology Co., Ltd.) containing $1 \mathrm{mM}$ phenylmethylsulfonyl fluoride. The cell extracts were centrifuged at $12,000 \mathrm{xg}$ for $5 \mathrm{~min}$ at $4^{\circ} \mathrm{C}$, and the supernatants were collected. The protein concentrations were determined using a bicinchoninic acid protein concentration detection kit (Biyuntian Bio-Technology, Co., Ltd.). Total protein lysates (20 $\mu \mathrm{g}$ protein) were separated by $12 \%$ SDS-PAGE, and transferred to $0.22-\mu \mathrm{M}$ nitrocellulose membranes, which were blocked in Tris-buffered saline Tween $(0.1 \%$ Tween-20) containing 5\% non-fat dry milk for $1 \mathrm{~h}$ and incubated overnight at $4^{\circ} \mathrm{C}$ with primary antibodies against caspase-3 (cat. no. 19677-1-AP), poly(ADP-ribose) polymerase (PARP; cat. no. 66520-1-Ig), P53 (cat. no. 60283-1-Ig), BAX (cat. no. 60267-1-Ig), Bcl-2 (cat. no. 60178-1-Ig), BCL-xL 

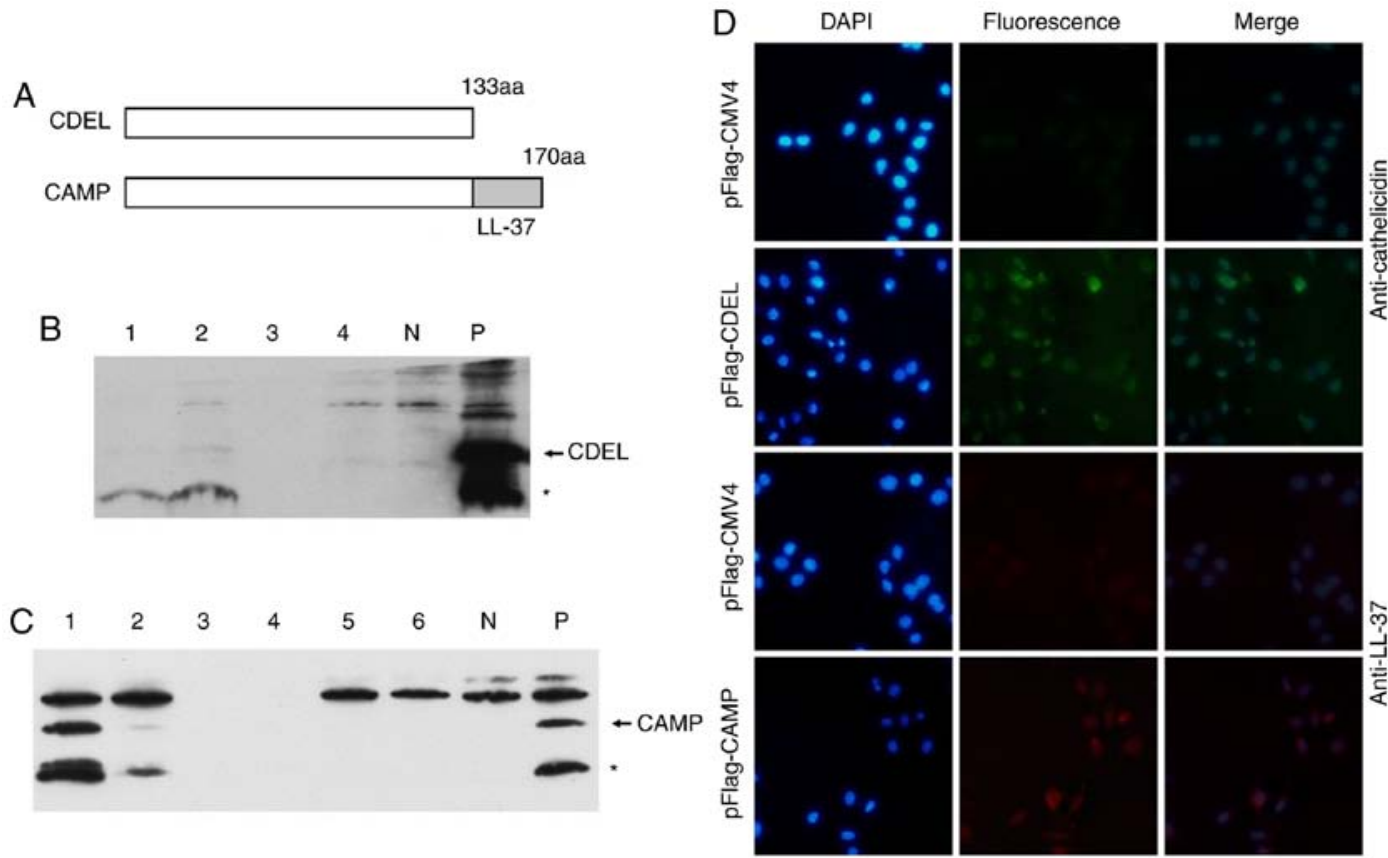

Figure 1. Generation of CDEL and CAMP stably transfected cells. (A) Structure diagram of CDEL and CAMP. (B) CDEL protein expression examined by western blot analysis using anti-cathelicidin antibody. Lane 1-4, protein extracts from different clones. KB cells were transiently transfected with pFlag-CMV4 (N) and pFlag-CDEL(P), respectively. (C) CAMP protein expression examined by western blot analysis using anti-LL-37 antibody. Lane 1-6, protein extracts from different clones. KB cells were transiently transfected with pFlag-CMV4 (N) and pFlag-CAMP (P), respectively. (D) Characterization of CDEL and CAMP stably transfected cells using immunofluorescence analysis. In the above two rows of pFlag-CMV4 and pFlag-CDEL, the anti-cathelicidin antibody was used as the primary antibody, and Alexa Fluor 594-conjugated goat anti-rabbit IgG antibody was used as the secondary fluorescent antibody. In the following two rows of pFlag-CMV4 and pFlag-CAMP, anti-LL-37 was used as the primary antibody, and Alexa Fluor 594-conjugated goat anti-mouse IgG was used as the secondary fluorescent antibody. Arrows indicate the target proteins, and asterisks indicate degraded target proteins. CDEL, LL-37 C-terminal deletion mutant.

(cat. no. 66020-1-Ig), cyclin B1 (cat. no. 55004-1-AP), PKR-like ER kinase (PERK; cat. no. 20582-1-AP), Akt (cat. no. 60203-2-Ig), phospho- (p-)Akt (cat. no. 66444-1-Ig), ERK (cat. no. 66192-1-Ig) (all from ProteinTech Group, Inc.), cleaved-caspase-3 (cat. no. 9661; Cell Signalling Technology, Inc.), cathelicidin (cat. no. ab69484; Abcam), p-ERK (cat. no. ab76299; Abcam), LL-37 (cat. no. sc-166770; Santa Cruz Biotechnology, Inc.) or $\beta$-actin (cat. no. TA-09; OriGene Technologies, Inc.) all at a dilution of 1:2,000. The membranes were washed and then incubated for $2 \mathrm{~h}$ at room temperature with horseradish peroxidase-conjugated goat anti-rabbit antibodies or goat anti-mouse antibodies (cat. nos. EM35111-01 and EM35110-01; EMAR Biotechnology) at a dilution of 1:3,000. The immunoreactions were visualized using Clarity ${ }^{\mathrm{TM}}$ Western ECL substrate (Bio-Rad Laboratories, Inc.) and exposed to Amersham Hyperfilm ECL film (Amersham, Cytiva). Protein bands were quantified by Quantity One version 4.6.3 (Bio-Rad Laboratories, Inc.).

RNA-seq. Total cellular RNA was extracted using TRIzol ${ }^{\circledR}$ reagent (Thermo Fisher Scientific, Inc.). The RNA-Seq assay was performed (Novagene) as described previously (31). Briefly, mRNA-seq libraries were prepared using standard Illumina protocols, and the mRNA libraries were then sequenced on an Illumina HiSeq 2000 platform using a 101-bp paired-end sequencing strategy. A reads per kilobase transcriptome per million reads method was used to calculate expression levels of genes (32). Differential expression analysis of two groups was performed using the DESeq R package version 1.10.1 (33). KOBAS version 2.0 software (http://kobas.cbi.pku.edu.cn) was used to examine the statistical enrichment of differentially expressed genes in Kyoto Encyclopaedia of Genes and Genomes (KEGG) pathways.

Statistical analysis. A total of 3-6 independent experiments were performed in the present study. Statistical analysis was performed using SPSS version 19.0 software (IBM Corp.). A one-way ANOVA followed by a Bonferroni's post hoc test was used for multiple comparisons. $\mathrm{P}<0.05$ was considered to indicate a statistically significant difference.

\section{Results}

Generation of stable expression cell lines. To explore the roles and mechanisms of human CAMP in OSCC cells, a full-length CAMP and a LL-37 CDEL were used (Fig. 1A). To generate stably expressing cell lines, either CDEL or CAMP ORFs were cloned into the eukaryotic expression vector pFlag-CMV4, and these vectors were then transfected into HSC-3 cells after selecting for transfected cells using G418, different monoclonal cells were obtained by gradient dilution. Monoclonal cells which stably expressed either CDEL or CAMP were screened by western blot analysis after expanding the cultures (Fig. 1B and C).

The results of the immunofluorescence analysis revealed that there was no significant fluorescence of pFlag-CMV4-transfected cells (negative control), and notable green fluorescence was observed in the pFlag-CDEL-transfected cells (Fig. 1D). The results also illustrated that no significant fluorescence was observed in the pFlag-CMV4-transfected cells 

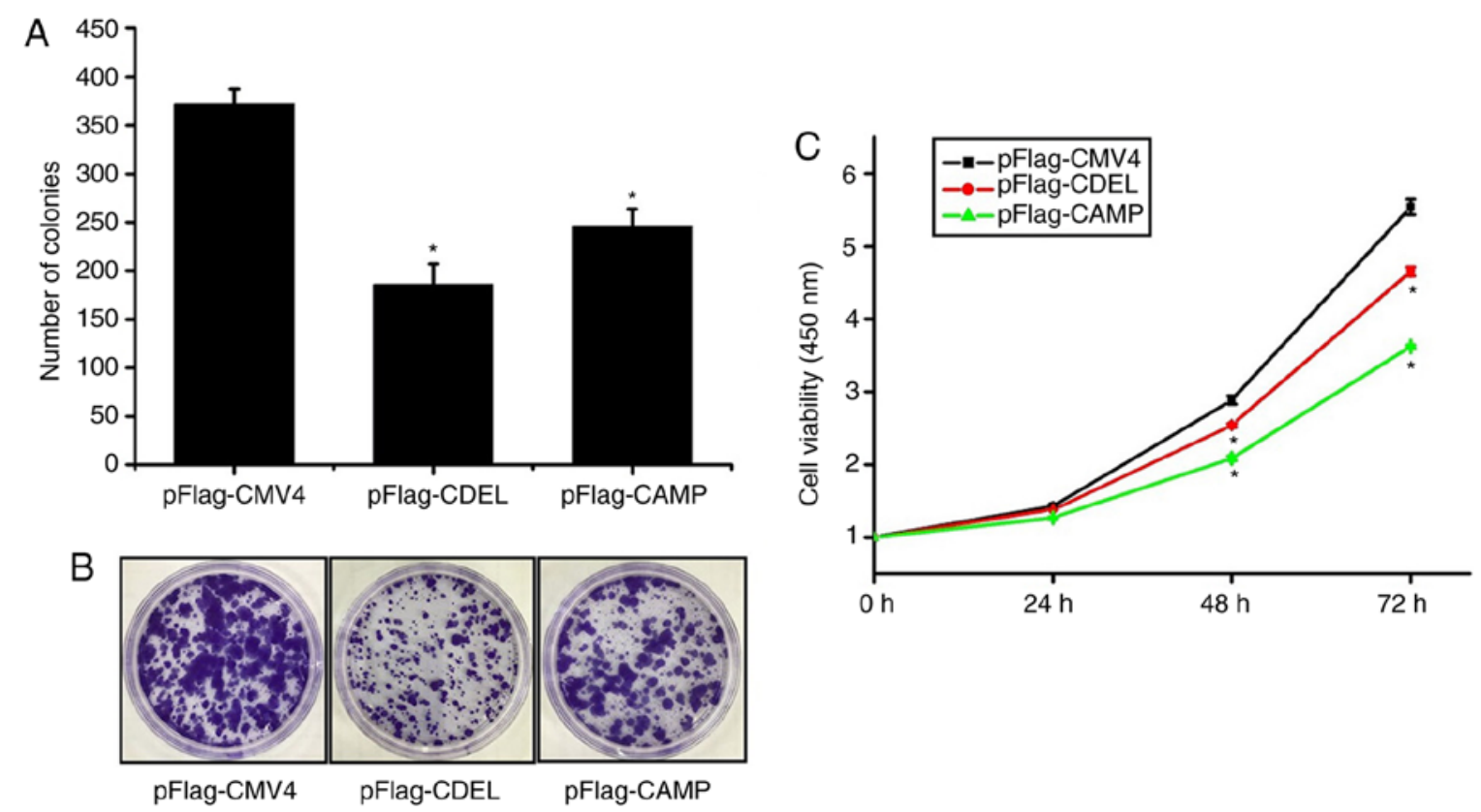

Figure 2. Either CDEL or CAMP overexpression inhibits HSC-3 cell proliferation. (A) Cells were plated on $10 \mathrm{~cm}$ plates at a density of $1 \times 10^{3}$ cells/well. After 12 days, the cell number was counted. (B) Representative images of crystal violet staining. (C) Viability of either CDEL or CAMP-transfected HSC-3 cells measured using a CCK-8 assay. CCK-8 assays were performed at 0, 24, 48 and $72 \mathrm{~h}$. Data are presented as the mean \pm standard error of the mean of 3-6 repeats. ${ }^{*} \mathrm{P}<0.05$ vs. control. CCK-8, Cell Counting Kit-8; CDEL, LL-37 C-terminal deletion mutant.

(negative control), whereas red fluorescence was observed in the pFlag-CAMP-transfected cells (Fig. 1D). These results indicated that HSC-3 cells with CDEL and CAMP stable expression were successfully generated.

CDEL and CAMP stable expression in HSC-3 cells inhibits colony formation and cell proliferation. The results of the colony-formation assay revealed that the number of cell clones of either CDEL or CAMP stably expressing HSC-3 cells was significantly lower than that of HSC-3 cells stably transfected with the empty vector (Fig. 2A and B). This result demonstrated that either CDEL or CAMP stable expression in HSC-3 cells inhibited the colony formation ability compared with the control.

The results of the CCK-8 assay showed that the viability of either the CDEL or CAMP stably expressing HSC-3 cells was lower than that of the empty vector-transfected HSC-3 cells after 24,48 and $72 \mathrm{~h}$, respectively (Fig. 2C). These results indicated that the proliferation and viability of either the CDEL or CAMP stably expressing HSC-3 cells were significantly lower than that of the controls.

CDEL and CAMP stable expression in HSC-3 cells inhibits cell migration and invasion. The results of the wound healing assay revealed that the cell-free area of either the CDEL or CAMP stably expressing HSC-3 cells was significantly larger than that of the controls at 12,24 and $36 \mathrm{~h}$, respectively (Fig. 3A). This result indicated that the migratory ability of either the CDEL or CAMP stably expressing HSC-3 cells was significantly lower than that of the controls.

The results of the Transwell invasion assay revealed that the number of invaded CDEL or CAMP stably expressing HSC-3 cells were significantly lower than those of the controls (Fig. 3B). This result indicates that the invasive ability of either the CDEL or CAMP stably expressing HSC-3 cells is decreased significantly compared with that of the controls.

Either CDEL or CAMP overexpression triggers caspase-3 mediated apoptosis of HSC-3 cells. To explore the mechanisms of either CDEL or CAMP in HSC-3 cells, western blot analysis was performed. The results revealed that the expression levels of cleaved caspase-3 and cleaved PARP were significantly upregulated, whereas the expression levels of caspase- 3 were downregulated in both the CDEL- or CAMP-transfected cells (Fig. 4A). Moreover, the expression levels of the apoptosis-promoting proteins, P53 and BAX, in either the CDEL- or CAMP-transfected cells were significantly upregulated, whereas the expression levels of the apoptosis-inhibiting proteins, $\mathrm{Bcl}-2$ and Bcl-xL, in either the CDEL- or CAMP-transfected cells were significantly downregulated (Fig. 4A). These results indicated that CDEL or CAMP overexpression induced caspase-3 mediated apoptosis via the P53-Bcl-2/BAX signalling pathway in OSCC HSC-3 cells. Taken together, these results suggested that either CDEL or CAMP overexpression induces caspase-3 mediated apoptosis of HSC-3 cells and exerts a tumour-suppressive effect on stably transfected HSC-3 cells.

The results demonstrated that the expression levels of the cell cycle-related proteins, Cyclin B1 or PERK, were significantly upregulated in the CAMP-, but not in the CDEL-transfected cells (Fig. 4B), which suggested that the possible mechanisms by which CDEL and CAMP overexpression in cells affected the cell cycle was different. Moreover, the p-Akt/total Akt and p-ERKs/total ERKs ratios were notably decreased in both the CDEL- or CAMP-transfected cells (Fig. 4C).

Comparison of transcriptional profiles. To further explore other possible molecular mechanisms of either CDEL or CAMP in OSCC HSC-3 cells, the transcriptomes of three 

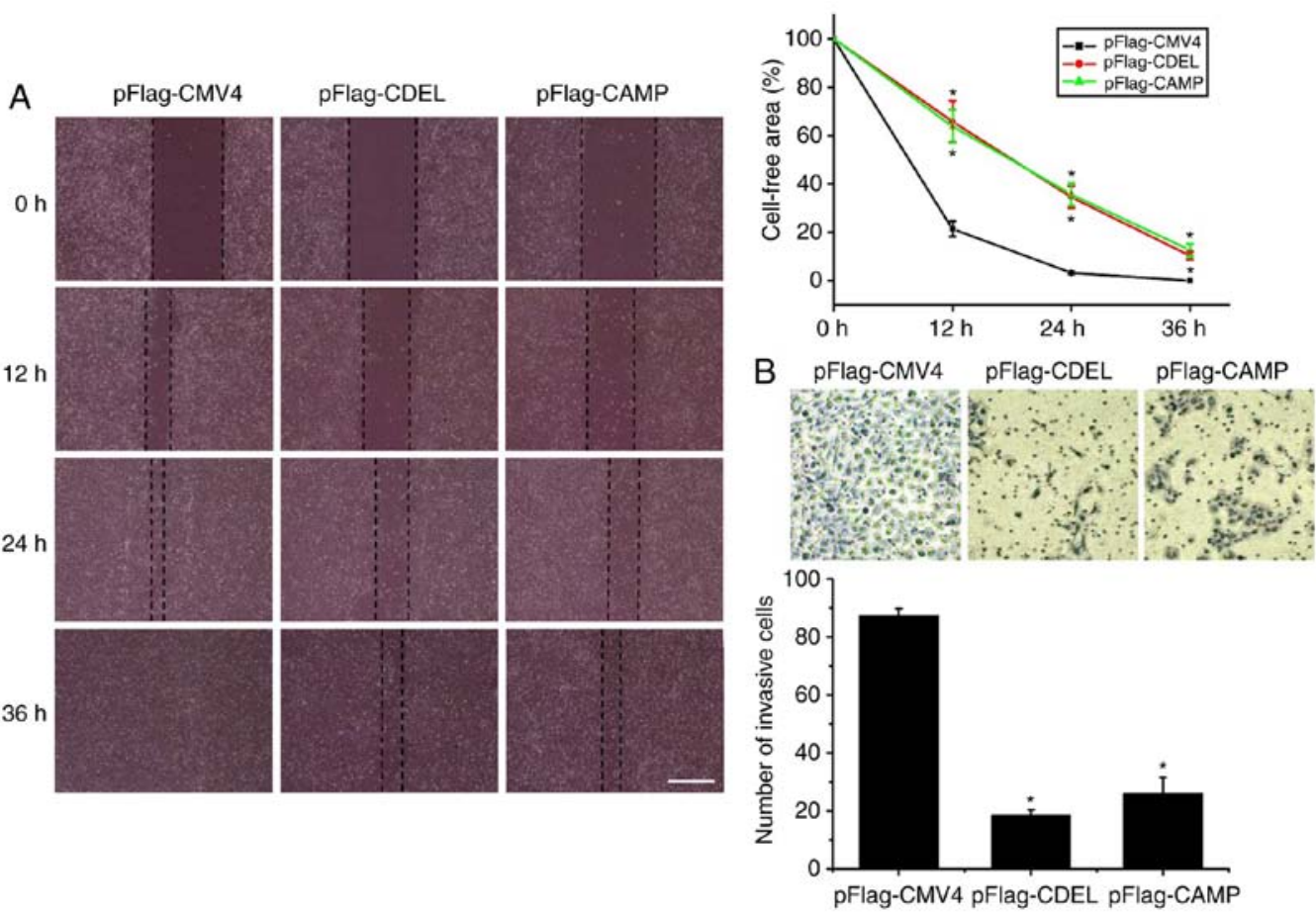

Figure 3. Both CDEL and CAMP overexpression inhibits the migration and invasion of HSC-3 cells. (A) Wound healing expressed as a function of time, observed for up to $36 \mathrm{~h}$. Quantitative analysis of the cell-free area at a different time. (B) Transwell assays were used to analyse the invasive ability of the HSC-3 cells. Representative images of stained cells are shown. Scale bar, $400 \mu \mathrm{m}$. Quantitative analysis of the invaded cells was counted. Data are presented as the mean \pm standard error of the mean of 3-6 repeats. "P<0.05 vs. control. CDEL, LL-37 C-terminal deletion mutant.

A pFlag- pFlag- pFlag-
$\mathrm{kDa}$ CMV4 CDEL CAMP

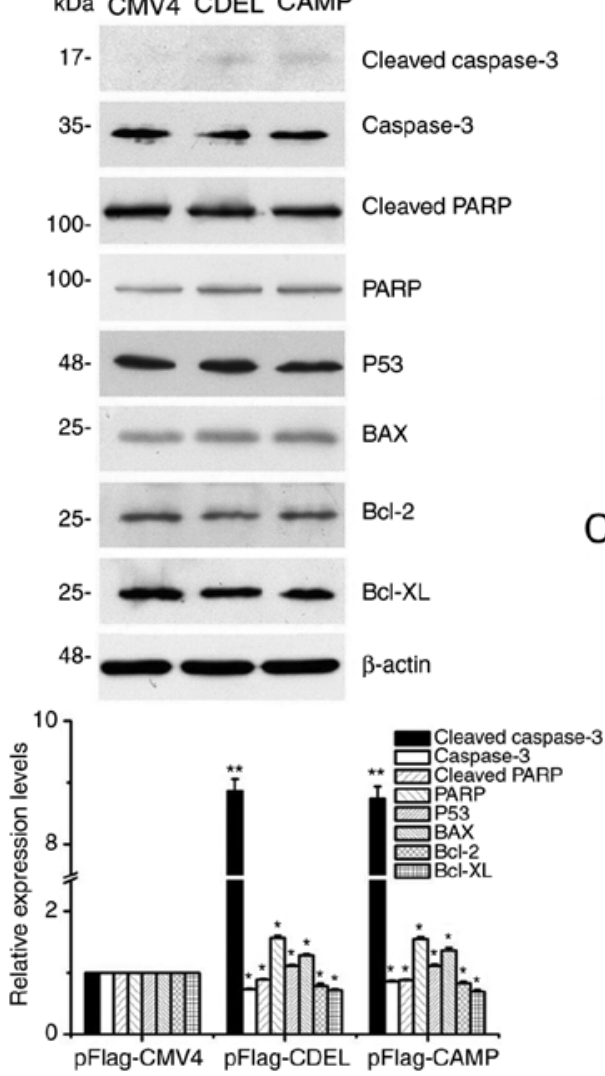

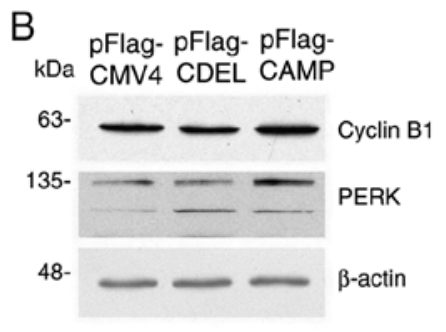
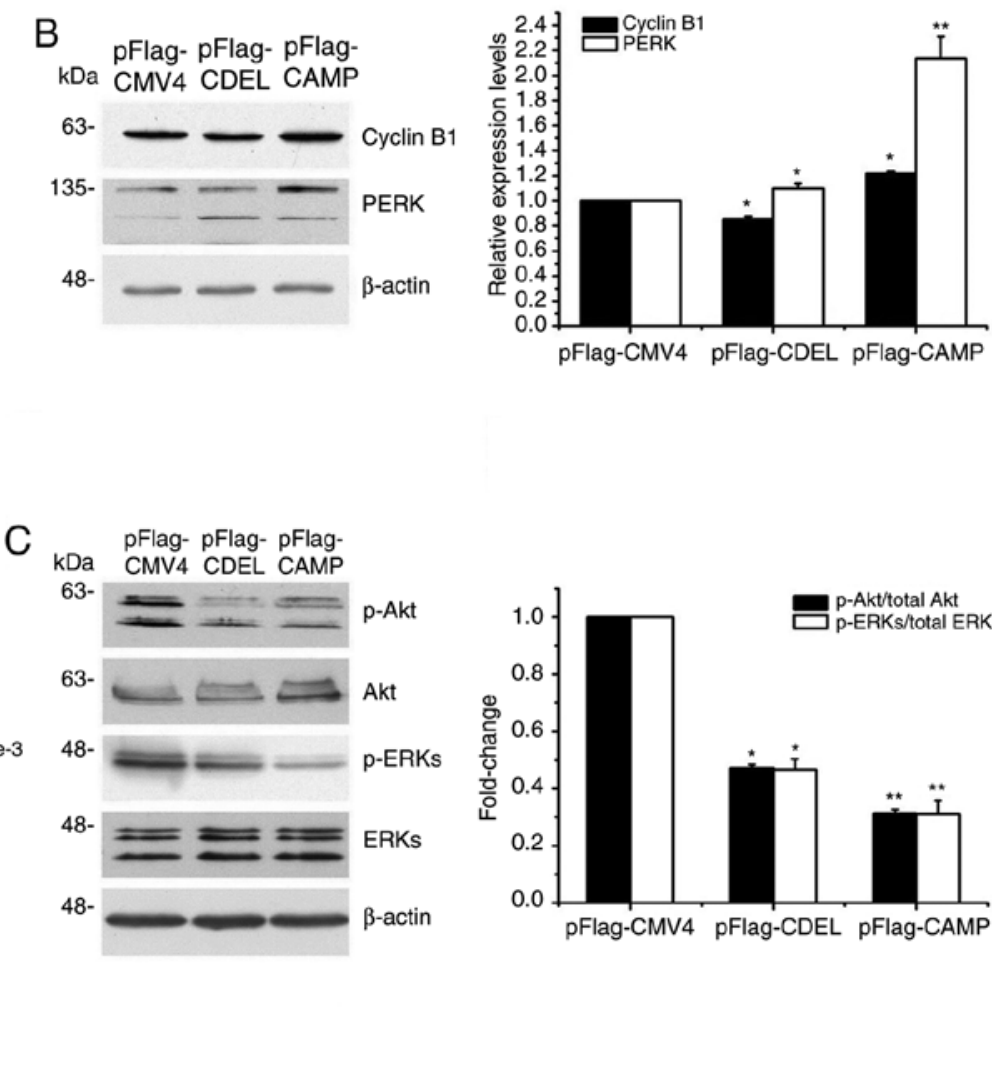

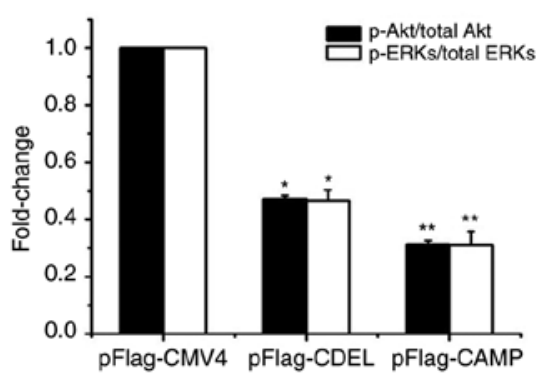

Figure 4. CDEL, as well as CAMP overexpression can induce caspase-3 mediated apoptosis. (A) Expression analysis of apoptosis-related proteins, including cleaved-caspase-3, caspase-3, cleaved-PARP, p53, BAX, Bcl-2 and Bcl-xL using western blot analysis. (B) Expression analysis of cell cycle-related proteins, including Cyclin B1 and PERK using western blot analysis. (C) Expression analysis of phosphorylated and total Akt and ERK. The ratio of p-Akt/total Akt and p-ERKs/total ERKs was quantified. Representative blots of independent experiments are shown. The bands were quantified relative to $\beta$-actin. ${ }^{*} \mathrm{P}<0.05$, ${ }^{* *} \mathrm{P}<0.01$ vs. control. CDEL, LL-37 C-terminal deletion mutant; PARP, poly(ADP-ribose) polymerase; PERK, PKR-like ER kinase; p-, phospho. 

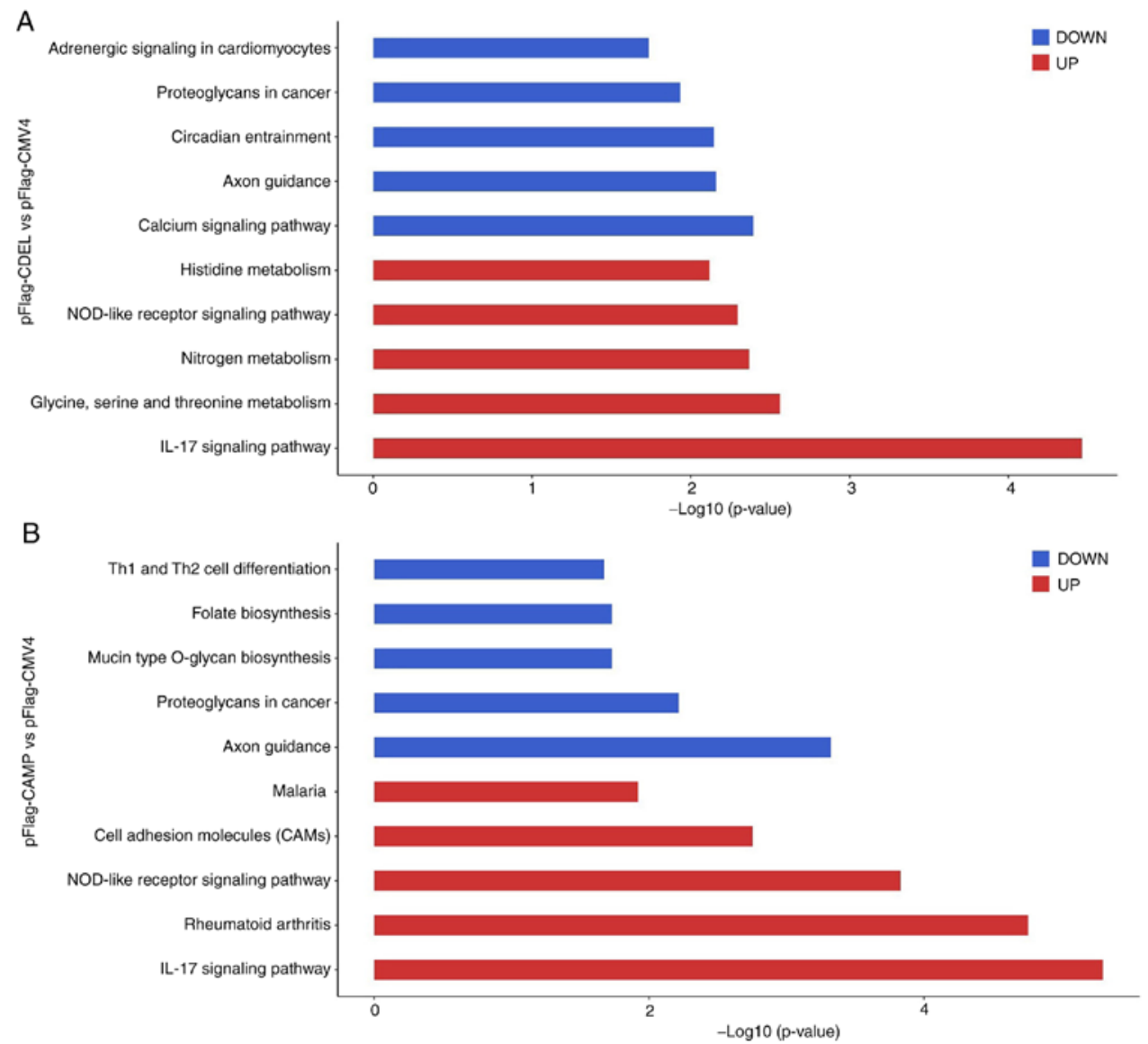

Figure 5. KEGG enrichment analysis of differentially expressed genes. (A) Comparison of pFlag-CDEL and pFlag-CMV4 stably transfected HSC-3 cells (B) Comparison of pFlag-CAMP and pFlag-CMV4 stably transfected HSC-3 cells. The top downregulated genes are illustrated in blue and top upregulated genes are illustrated in red. CDEL, LL-37 C-terminal deletion mutant; NOD, nucleotide oligomerization domain.

stable cell lines were compared. The comparison of the CDEL-transfected HSC-3 cells with the controls revealed that the top upregulated genes were involved in the enrichment of KEGG processes associated with IL-17 signalling pathway, glycine, serine and threonine metabolism, nitrogen metabolism, nucleotide oligomerization domain (NOD)-like receptor signalling pathway and histidine metabolism; whereas the top downregulated genes were involved in the calcium signalling pathway, axon guidance circadian entrainment (Fig. 5A). Similarly, the comparison of the CAMP-transfected HSC-3 cells with the controls revealed that top upregulated genes were involved in the IL-17 signalling pathway, rheumatoid arthritis and NOD-like receptor signalling pathway; whereas the top downregulated genes were involved in axon guidance, proteoglycans in cancer and mucin type O-glycan biosynthesis (Fig. 5B). Each comparison indicated that the top upregulated genes were involved in IL-17-dependent and NOD-like receptor signalling pathways, which are closely associated with the initiation and progression of cancer $(34,35)$.

\section{Discussion}

Previous studies have demonstrated the roles and mechanisms of human CAMP/LL-37 involvement in various types of cancer (6-9). A previous study also indicated that human CAMP/LL-37 was expressed at low levels in OSCC, where it may exert an inhibitory effect on development, and DNA methylation may serve a role in OSCC by directly downregulating CAMP gene promoter activity (24). Human CAMP/LL-37 has multiple roles and mechanisms in various cancer cells $(6-21,36)$. Both tumorigenic and anti-cancer effects induced by CAMP/LL-37 have been reported in various types of cancer, and the combined effects of those involved mechanisms determine the final effect $(6,9)$. The possible mechanisms of human CAMP/LL-37 in the different OSCC cells have also been explored previously $(22,23)$. However, the detailed roles and underlying mechanisms of human CAMP/LL-37 in OSCC cells remains unknown, to the best of our knowledge. The present study provides an improved understanding of the roles and mechanisms of human CAMP/LL-37 in OSCC.

The overexpression of either CDEL or CAMP in OSCC HSC-3 cells reduced colony-formation, proliferation and viability, as well as their migratory and invasive ability. The results revealed that the overexpression of either CDEL or CAMP also promoted the apoptosis of HSC- 3 cells, and induced caspase-3 mediated apoptosis via the P53-Bcl-2/BAX signalling pathway, so as to exert an anticancer effect on OSCC HSC-3 cells. It has been previously shown that human CAMP/LL-37 and its analogues induce caspase-independent apoptosis in colon cancer $(18,19)$ and haematological malignancies (20). The results of the present study also showed that the overexpression of CDEL exerted a more potent inhibitory effect on colony formation, migration and invasion ability 
compared with that of CAMP. Therefore, it seems that the LL-37 fragment may exert an opposing effect to that of the CDEL fragment. The anti-cancer effects of cathelicidin LL-37 in gastric cancer has been proposed to involve G0/G1 phase cell cycle arrest (17). The cell cycle arrest in $\mathrm{G} 2 / \mathrm{M}$ via $\mathrm{p} 21$ activation leads to the anti-proliferative effects of cathelicidin LL-37 in colon cancer cells (19,37). The results of the present study also demonstrated that the levels of cell cycle-related proteins, cyclin B1 and PERK, were significantly upregulated in the CAMP, but not in the CDEL-transfected cells. These results suggested that the potential mechanism of anticancer effects of CAMP but not CDEL on OSCC HSC-3 cells may be attributed to cell cycle regulation. The Akt and ERK signalling pathways are associated with proliferation, differentiation, migration, invasion and apoptosis in cancer cells $(38,39)$. The decreased p-Akt/total Akt and p-ERKs/total ERKs ratios suggested that the Akt and ERK signalling pathway may be involved in the tumour-suppressive function of either CDEL or CAMP in HSC-3 cells (Fig. 4C). Conversely, the results also suggested that the C-terminal of CAMP (LL-37) may exert marked effects on OSCC cells, and HSC-3 cells stimulated by artificially synthesized LL-37, thus, small peptides may assist in examining the effects of LL-37 extracellular treatment and its mechanism of action.

IL-17-dependent and NOD-like receptor signalling pathways have significant roles in inflammatory diseases and cancer $(34,35)$. Transcriptome analysis revealed differences between CDEL or CAMP-transfected HSC-3 cells when compared with the control. Upregulated genes involved in the IL-17-dependent and NOD-like receptor signalling pathways were observed for each comparison, whereas a number of differentially expressed genes were also observed for each comparison. However, the detailed molecular mechanism remains to be further clarified.

In the present study, determination of the protein expression of pFlag-CDEL stably-transfected HSC-3 cells using a Flag antibody was attempted; however, the results were not satisfactory (data not shown). These results were possibly due to the fact that Flag at the N-terminal of the protein was cut off during the expression or preparation of cell-lysed protein samples in HSC-3 cells. Therefore, various cathelicidin antibodies were used to detect the expression of CDEL in HSC-3 cells and it was found that the expression of CDEL in HSC-3 cells could be effectively detected with an Abcam anti-cathelicidin antibody (the epitope is located at 50-100 of the CAMP protein). However, it should be noted that the results of the present study were only observed in OSCC HSC-3 cells and the effects may differ in other cells, such as gingival cells. In addition, the results of the present study are based upon data obtained in vitro and examination using signal inhibitors should also be performed in subsequent studies.

In conclusion, the present study successfully established HSC-3 cell lines stably expressing either CDEL or CAMP, and examined the roles and mechanisms of CDEL or CAMP in these cells. To the best of our knowledge, the present study is the first to show that CAMP/LL-37 may act as a tumour suppressor in OSCC cells, and the underlying mechanism may include the induction of caspase-3 dependent apoptosis via the P53-Bcl-2/BAX signalling pathway. Further studies using other OSCC cells and in vivo studies will undoubtedly deepen our understanding of its roles and mechanisms in the initiation and progression of OSCC.

\section{Acknowledgements}

Not applicable.

\section{Funding}

This study was supported by grants from the National Natural Science Foundation of China (grant no. 81760490) and Natural Science Foundation of Guangxi (grant no. 2017GXNSFAA198239).

\section{Availability of data and materials}

The datasets used and/or analysed during the current study are available from the corresponding author on reasonable request.

\section{Authors' contributions}

$\mathrm{XC}, \mathrm{SJ}, \mathrm{JS}, \mathrm{YG}$ and XZo made substantial contributions to the conception and design of the study. XZh and XW contributed to data analysis and interpretation. All authors read and approved the final manuscript.

\section{Ethics approval and consent to participate}

Not applicable.

\section{Patient consent for publication}

Not applicable.

\section{Competing interests}

The authors declare that they have no competing interests.

\section{References}

1. Alagarasu K, Patil PS, Shil P, Seervi M, Kakade MB, Tillu H and Salunke A: In-vitro effect of human cathelicidin antimicrobial peptide LL-37 on dengue virus type 2. Peptides 92: 23-30, 2017.

2. Majewski K, Żelechowska P and Brzezińska-Błaszczyk E: Circulating cathelicidin LL-37 in adult patients with pulmonary infectious diseases. Clin Invest Med 40: E34-E39, 2017.

3. Tsai PW, Yang CY, Chang HT and Lan CY: Human antimicrobial peptide LL-37 inhibits adhesion of Candida albicans by interacting with yeast cell-wall carbohydrates. PLoS One 6: e17755, 2011.

4. Polcyn-Adamczak M and Niemir ZI: Cathelicidin - Its structure, function and the role in autoimmune diseases. Adv Cell Biol 4: 83-96, 2014.

5. Kahlenberg JM and Kaplan MJ: Little peptide, big effects: The role of LL-37 in inflammation and autoimmune disease. J Immunol 191: 4895-4901, 2013.

6. Piktel E, Niemirowicz K, Wnorowska U, Wątek M, Wollny T, Głuszek K, Góźdź S, Levental I and Bucki R: The role of cathelicidin LL-37 in cancer development. Arch Immunol Ther Exp (Warsz) 64: 33-46, 2016.

7. Wu WK, Wang G, Coffelt SB, Betancourt AM, Lee CW, Fan D, Wu K, Yu J, Sung JJ and Cho CH: Emerging roles of the host defense peptide LL-37 in human cancer and its potential therapeutic applications. Int J Cancer 127: 1741-1747, 2010. 
8. Kuroda K, Okumura K, Isogai $\mathrm{H}$ and Isogai E: The human cathelicidin antimicrobial peptide LL-37 and mimics are potential anticancer drugs. Front Oncol 5: 144, 2015.

9. Chen X, Zou X, Qi G, Tang Y, Guo Y, Si J and Liang L: Roles and mechanism of human cathelicidin LL-37 in cancer. Cell Physiol Biochem 47: 1060-1073, 2018.

10. Carmona FJ, Montemurro F, Kannan S, Rossi V, Verma C, Baselga $\mathbf{J}$ and Scaltriti M: AKT signaling in ERBB2-amplified breast cancer. Pharmacol Ther 158: 63-70, 2016.

11. Li D, Wang X, Wu JL, Quan WQ, Ma L, Yang F, Wu KY and Wan HY: Tumor-produced versican V1 enhances hCAP18/LL-37 expression in macrophages through activation of TLR 2 and vitamin D3 signaling to promote ovarian cancer progression in vitro. PLoS One 8: e56616, 2013.

12. von Haussen J, Koczulla R, Shaykhiev R, Herr C, Pinkenburg O, Reimer D, Wiewrodt R, Biesterfeld S, Aigner A, Czubayko F, et al: The host defence peptide LL-37/hCAP-18 is a growth factor for lung cancer cells. Lung Cancer 59: 12-23, 2008.

13. Hensel JA, Chanda D, Kumar S, Sawant A, Grizzle WE, Siegal GP and Ponnazhagan S: LL-37 as a therapeutic target for late stage prostate cancer. Prostate 71: 659-670, 2011

14. Sainz B Jr, Alcala S, Garcia E, Sanchez-Ripoll Y, Azevedo MM, Cioffi M, Tatari M, Miranda-Lorenzo I, Hidalgo M, Gomez-Lopez G, et al: Microenvironmental hCAP-18/LL-37 promotes pancreatic ductal adenocarcinoma by activating its cancer stem cell compartment. Gut 64: 1921-1935, 2015.

15. Muñoz M, Craske M, Severino $P$, de Lima TM, Labhart $P$, Chammas R, Velasco IT, Machado MC, Egan B, Nakaya HI, et al: Antimicrobial peptide LL-37 participates in the transcriptional regulation of melanoma cells. J Cancer 7: 2341-2345, 2016.

16. Wang W, Zheng Y, Jia J, Li C, Duan Q, Li R, Wang X, Shao Y, Chen C and Yan H: Antimicrobial peptide LL-37 promotes the viability and invasion of skin squamous cell carcinoma by upregulating YB-1. Exp Ther Med 14: 499-506, 2017.

17. 17. Wu WK, Sung JJ, To KF, Yu L, Li HT, Li ZJ, Chu KM, $\mathrm{Yu} \mathrm{J}$ and Cho $\mathrm{CH}$ : The host defense peptide LL-37 activates the tumor-suppressing bone morphogenetic protein signaling via inhibition of proteasome in gastric cancer cells. J Cell Physiol 223: 178-186, 2010.

18. Ren SX, Cheng AS, To KF, Tong JH, Li MS, Shen J, Wong CC Zhang L, Chan RL, Wang XJ, et al: Host immune defense peptide LL-37 activates caspase-independent apoptosis and suppresses colon cancer. Cancer Res 72: 6512-6523, 2012.

19. Kuroda K, Fukuda T, Krstic-Demonacos M, Demonacos C, Okumura K, Isogai H, Hayashi M, Saito K and Isogai E: miR-663a regulates growth of colon cancer cells, after administration of antimicrobial peptides, by targeting CXCR4-p21 pathway. BMC Cancer 17: 33, 2017.

20. Mader JS, Mookherjee N, Hancock RE and Bleackley RC: The human host defense peptide LL-37 induces apoptosis in a calpainand apoptosis-inducing factor-dependent manner involving Bax activity. Mol Cancer Res 7: 689-702, 2009.

21. An LL, Ma XT, Yang YH, Lin YM, Song YH and Wu KF. Marked reduction of LL-37/hCAP-18, an antimicrobial peptide, in patients with acute myeloid leukemia. Int J Hematol 81: 45-47, 2005.

22. Okumura K, Itoh A, Isogai E, Hirose K, Hosokawa Y, Abiko Y, Shibata T, Hirata $M$ and Isogai $H$ : C-terminal domain of human CAP18 antimicrobial peptide induces apoptosis in oral squamous cell carcinoma SAS-H1 cells. Cancer Lett 212: 185-194, 2004

23. Acil Y, Torz K, Gülses A, Wieker H, Gerle M, Purcz N, Will OM, Eduard Meyer J and Wiltfang J: An experimental study on antitumoral effects of KI-21-3, a synthetic fragment of antimicrobial peptide LL-37, on oral squamous cell carcinoma. J Craniomaxillofac Surg 46: 1586-1592, 2018.
24. Chen X, Qi G, Qin M, Zou Y, Zhong K, Tang Y, Guo Y, Jiang X, Liang L and Zou X: DNA methylation directly downregulates human cathelicidin antimicrobial peptide gene (CAMP) promoter activity. Oncotarget 8: 27943-27952, 2017.

25. Väyrynen O, Åström P, Nyberg P, Alahuhta I, Pirilä E, Vilen ST, Aikio M, Heljasvaara R, Risteli M, Sutinen M, et al: Matrix metalloproteinase 9 inhibits the motility of highly aggressive HSC-3 oral squamous cell carcinoma cells. Exp Cell Res 376: 18-26, 2019.

26. Chang WL, Cheng FC, Wang SP, Chou ST and Shih Y: Cinnamomum cassia essential oil and its major constituent cinnamaldehyde induced cell cycle arrest and apoptosis in human oral squamous cell carcinoma HSC-3 cells. Environ Toxicol 32: 456-468, 2017.

27. Araújo T, Khayat A, Quintana L, Calcagno D, Mourão R, Modesto A, Paiva J, Lima A, Moreira F, Oliveira E, et al: Piwi like RNA-mediated gene silencing 1 gene as a possible major player in gastric cancer. World J Gastroenterol 24: 5338-5350, 2018.

28. Tang B, Qi G, Tang F, Yuan S, Wang Z, Liang X, Li B, Yu S, Liu J, Huang Q, et al: Aberrant JMJD3 expression upregulates slug to promote migration, invasion, and stem cell-like behaviors in hepatocellular carcinoma. Cancer Res 76: 6520-6532, 2016.

29. Qi G, Kudo Y, Tang B, Liu T, Jin S, Liu J, Zuo X, Mi S, Shao W, Ma X, et al: PARP6 acts as a tumor suppressor via downregulating Survivin expression in colorectal cancer. Oncotarget 7: 18812-18824, 2016.

30. Zhao JG, Wang JF, Feng JF, Jin XY and Ye WL: HHIP overexpression inhibits the proliferation, migration and invasion of non-small cell lung cancer. PLoS One 14: e0225755, 2019.

31. Duan Q, Xiao Y, Zhu L, Liu Z, Mao X, Zhou Z, Liao C, Cai J, Huang F, Liu Z, et al: BET bromodomain is a novel regulator of TAZ and its activity. Biochim Biophys Acta 1859: 1527-1537, 2016.

32. Mortazavi A, Williams BA, McCue K, Schaeffer L and Wold B: Mapping and quantifying mammalian transcriptomes by RNA-Seq. Nat Methods 5: 621-628, 2008.

33. Anders $S$ and Huber W: Differential expression analysis for sequence count data. Genome Biol 11: R106, 2010.

34. Vonderheide RH: Tumor-promoting inflammatory networks in pancreatic neoplasia: Another reason to loathe Kras. Cancer Cell 25: 553-554, 2014.

35. Kim YK, Shin JS and Nahm MH: NOD-like receptors in infection, immunity, and diseases. Yonsei Med J 57: 5-14, 2016.

36. Tuomela JM, Sandholm JA, Kaakinen M, Hayden KL, Haapasaari KM, Jukkola-Vuorinen A, Kauppila JH,Lehenkari PP, Harris KW, Graves DE, et al: Telomeric G-quadruplex-forming DNA fragments induce TLR9-mediated and LL-37-regulated invasion in breast cancer cells in vitro. Breast Cancer Res Treat 155: 261-271, 2016.

37. Cheng M, Ho S, Yoo JH, Tran DH, Bakirtzi K, Su B, Tran DH, Kubota Y, Ichikawa R and Koon HW: Cathelicidin suppresses colon cancer development by inhibition of cancer associated fibroblasts. Clin Exp Gastroenterol 8: 13-29, 2014.

38. Martini M, De Santis MC, Braccini L, Gulluni F and Hirsch E: PI3K/AKT signaling pathway and cancer: An updated review. Ann Med 46: 372-383, 2014.

39. Sun Y, Liu WZ, Liu T, Feng X, Yang N and Zhou HF: Signaling pathway of MAPK/ERK in cell proliferation, differentiation, migration, senescence and apoptosis. J Recept Signal Transduct Res 35: 600-604, 2015.

This work is licensed under a Creative Commons Attribution-NonCommercial-NoDerivatives 4.0 International (CC BY-NC-ND 4.0) License. 